\title{
Estudio sobre necesidades formativas en cuidados paliativos para atención primaria
}

\author{
Cristina Lasmarías, José Espinosa, Marisa Martínez-Muñoz, Ingrid Bullich, Eulalia Alburquerque, \\ Xavier Gómez-Batiste
}

Introducción. La estrategia en cuidados paliativos del Sistema Nacional de Salud del año 2007 determina establecer programas de formación continuada específica para los profesionales del sistema sanitario con el fin de que atiendan adecuadamente las necesidades de los pacientes en situación de enfermedad avanzada o terminal, y de su familia. Se propone conocer las necesidades en formación percibidas por los profesionales de atención primaria en Cataluña relacionadas con cuidados paliativos y sugerir enfoques formativos que impacten en el cuidado de pacientes comunitarios en situación de enfermedad avanzada.

Pacientes y métodos. Se llevó a cabo un estudio observacional, descriptivo, transversal, con metodología cualitativa y cuantitativa, que incluía: a) encuesta a directores de equipos de atención primaria, determinando necesidades y prioridades formativas en cuidados paliativos y viabilidad de los planes formativos; y b) grupos focales con profesionales y docentes expertos en cuidados paliativos y profesionales de atención primaria, identificando similitudes y diferencias en las necesidades de formación percibidas en cuidados paliativos.

Resultados. De la muestra de equipos de atención primaria (48,7\% de los existentes), el 52\% refiere tener formación básica; el 15\%, intermedia, y el 3\%, avanzada, con una cobertura global superior al $70 \%$ de profesionales de atención primaria. Se percibe la necesidad de formación en cuidados paliativos, que debería generarse sistemáticamente en el marco de programas institucionales, con temas como el control de síntomas, las habilidades comunicativas y la optimización de la coordinación asistencial.

Conclusión. Se detecta una baja demanda de formación en habilidades de evaluación, dilemas éticos, planificación de las decisiones anticipadas y manejo del paciente no oncológico, aspectos relevantes en la atención al paciente en situación de enfermedad avanzada.

Palabras clave. Atención primaria. Cuidados paliativos. Educación. Necesidades de formación.

\section{Studying the training needs in palliative care of primary care teams}

Introduction. The strategy in palliative care established in 2007 within the National Health Service was that it was necessary to develop continuous training programs specific for health-care professionals with the objective of providing appropriate care for patients with advanced illness and/or end-of-life status, together with their families. The study sought to assess the training needs in palliative care as perceived by the Catalan health-care professionals in primary care; and to suggest training objectives that can have a positive effect on the care of patients with advanced illness within the community.

Patients and methods. The study was observational, descriptive and cross-sectional. The qualitative and quantitative methodology included: a) questionnaire involving team leaders in primary care to determine the needs and training priorities in palliative care and the viability of the training schemes; b) focus groups involving health-care professionals and experts in palliative care, and primary care workers, to identify similarities and differences (percieved by the professionals) in the requirements of palliative care training.

Results. From the primary care teams sample (48.7\% of total), we recorded $52 \%$ as having basic training, $15 \%$ as intermediate level, and $3 \%$ as advanced. The overall coverage was $70 \%$ of primary care professionals. The need for training in palliative care is explicitly acknowledged, as is the need for the training to be systematically developed within institutional programs. The requirements worth noting are: follow-up of symptoms, ease of communications, and optimisation of health-care coordination/provision.

Conclusion. Other outstanding educational aspects to be necessary included, though not demanded, are: assessment skills, ethical dilemmas or advanced care planning.

Key words. Education. Palliative care. Primary care. Training needs.
Pla Director Sociosanitari. Departament de Salut de Catalunya (I. Bullich). Observatorio Qualy; Centro Colaborador de la OMS para Programas Públicos de Cuidados Paliativos, CCOMS-ICO; Institut Català d'Oncologia (C. Lasmarías, J. Espinosa, M. Martínez-Muñoz, X. GómezBatiste). Unidad de Formación y Docencia; Institut Català d'Oncologia (E. Alburquerque). L'Hospitalet de Llobregat, Barcelona, España.

Correspondencia: Cristina Lasmarías Martínez. Observatorio Qualy / CCOMS-ICO. Institut Català d'Oncologia. Granvia, 199-203. E-08908 L'Hospitalet de Llobregat (Barcelona).

E-mail:

clasmarias@iconcologia.net

Agradecimientos:

A todos los profesionales sanitarios participantes, por su colaboración, y al Departament de Sanitat de la Generalitat de Catalunya, por su apoyo.

Conflicto de intereses: No declarado.

Competing interests: None declared.

(c) 2013 FEM 


\section{Introducción}

La atención a pacientes en situación de enfermedad avanzada o terminal se ha desarrollado significativamente en las últimas décadas en todo el territorio español $[1,2]$. Cataluña fue pionera implementando un modelo de atención basado en la asistencia sanitaria integral que incluye el control de síntomas, el apoyo emocional y espiritual de los pacientes y sus familias, y la organización de los equipos asistenciales centrada en las necesidades de los pacientes [3]. A partir de 1995, el programa de cuidados paliativos de Cataluña se consideró programa piloto por la Organización Mundial de la Salud (OMS) [4-7].

Un pilar fundamental de este programa ha sido la capacitación en cuidados paliativos de los profesionales sanitarios acercando la formación actualizada a todos los profesionales implicados $[8,9]$. Esta formación es particularmente importante en el ámbito de la atención primaria. La prevalencia actual de pacientes crónicos avanzados con pronóstico de vida limitado en Cataluña requiere profesionales capaces de dar respuesta a las demandas y necesidades de los pacientes, especialmente en este ámbito asistencial y en situaciones de baja o media complejidad.

En este aspecto destaca la existencia de los Programas de Atención Domiciliaria Equipos de Soporte (PADES), equipos de referencia en cuidados paliativos, que proporcionan apoyo a los equipos de atención primaria (EAP) compartiendo casos y asesorando sobre la intervención clínica adecuada, principalmente en situaciones de complejidad asistencial.

La estrategia en cuidados paliativos del Sistema Nacional de Salud del año 2007 [10] recoge las recomendaciones de formación para la mejora de la disciplina en todos los ámbitos de atención, especialmente en atención primaria:

- Cada comunidad autónoma debe establecer un plan de formación continuada específico en cuidados paliativos para los profesionales del sistema sanitario de cada ámbito de atención sanitaria.

- Los planes de formación se realizarán en cualquier nivel, tras el análisis de las necesidades formativas de los profesionales implicados.

Los EAP presentan necesidades formativas variadas, acordes con la variabilidad en la casuística que atienden. Sin embargo, su formación en cuidados paliativos está parcialmente estandarizada en los planes de formación de los equipos y, generalmente, condicionada al interés individual, así como poco reconocida profesional y económicamente [11].
Conocer el nivel de formación en cuidados paliativos [12,13] de los profesionales y la importancia otorgada a estos conocimientos es clave para desarrollar un plan de formación adaptado a las necesidades de aquéllos [14], orientado a optimizar la calidad asistencial al final de la vida y a satisfacer las necesidades de los pacientes y sus cuidadores [15].

En este estudio nos proponemos conocer las necesidades y demandas en formación percibidas por los profesionales de atención primaria en Cataluña en relación con los cuidados paliativos y proponer iniciativas formativas que tengan un impacto positivo en la asistencia sanitaria a pacientes en situación de enfermedad avanzada en la comunidad.

\section{Pacientes y métodos}

\section{Tipo de estudio, lugar y periodo de realización}

Se realizó un estudio observacional, descriptivo y transversal a lo largo de los años 2008-2009 sobre una muestra de profesionales del ámbito de la dirección, del ámbito asistencial de atención primaria, de los equipos PADES de Cataluña y de docentes expertos en cuidados paliativos.

\section{Muestra y criterios de selección}

Se escogieron dos muestras diferentes, una para la parte cuantitativa y otra para la cualitativa, con grupos focales:

- Los directores de los equipos se seleccionaron a partir de un sistema aleatorizado y representativo de los 271 equipos de atención primaria del Institut Català de la Salut (ICS).

- Para los grupos focales, se seleccionaron médicos de familia (MF), diplomados de enfermería (DUE) y trabajadores sociales (TS) de atención primaria, con experiencia mínima asistencial de dos años en un EAP de Cataluña. Se excluyeron profesionales no asistenciales o que hubiesen cursado formación de maestría en cuidados paliativos, al considerarlo un criterio de especialización en la disciplina.

Respecto a los profesionales de PADES y expertos docentes, se incluyó a médicos especialistas (ME), DUE y TS con al menos dos años de experiencia profesional en el PADES y que desarrollasen actividades formativas, formales o informales, sobre cuidados paliativos.

Se incluyeron docentes adjuntos de universidades o centros formativos reconocidos. A todos se 
Tabla I. Ítems del cuestionario estructurado a directores de centros de atención primaria.

Número de profesionales sanitarios: médicos de familia, enfermeras, trabajadores sociales, médicos residentes

Nivel de formación acreditada por los profesionales (avanzada, intermedia, básica) en cuidados paliativos

Actividades de formación relacionadas con cuidados paliativos realizadas en 2007

Necesidades de formación percibidas, factibilidad en la introducción de formación en sus programas formativos y demanda que los profesionales de los equipos hacen de ella (escala tipo Likert de 0 a 5 , siendo 0 el menor y 5 el mayor valor)

Prioridades formativas en aspectos clínicos y organizativos relacionados con su práctica habitual (categoría de priorización de 1 a 5, siendo 1 el más prioritario y el 5 el menos prioritario).

les solicitó el consentimiento para la participación y tratamiento confidencial de los datos.

Las técnicas utilizadas fueron:

- Cuantitativa. Se realizó una encuesta a través de un cuestionario estructurado a los directores de centros de atención primaria seleccionados, recogiendo los ítems descritos en la tabla I.

- Cualitativa. Se convocaron cuatro grupos focales compuestos por: 10 expertos docentes de entidades proveedoras de formación de cuidados paliativos (universidades, institutos de estudios, centros de coordinación de residentes, sociedades científicas), 12 profesionales de PADES representativos del territorio catalán y diferentes disciplinas (asistieron seis), y 12 profesionales de atención primaria, cuya selección aleatoria se basó en un criterio de representatividad geográfica, de entorno urbano, metropolitano y rural y de presencia multidisciplinar (asistieron 10 profesionales).

\section{Análisis estadístico}

Se realizó un análisis simple, descriptivo y de síntesis de los datos obtenidos de los cuestionarios y se tuvieron en cuenta:

- Variables cuantitativas: número total de EAP de Cataluña, número total de participantes y por especialidad, nivel de formación total (Tabla II) y por especialidad, y actividades formativas realizadas a lo largo de 2007.

- Variables cualitativas: escala aditiva tipo Likert de necesidad, demanda y factibilidad de intro-
Tabla II. Descripción de niveles de formación.

Formación de nivel básico

Realización de cursos básicos de cuidados paliativos, con una duración de 20-40 h. Contenidos: aspectos físicos (procesos de enfermedad, control de síntomas, farmacología y urgencias), psicosociales (relaciones familiares y sociales, comunicación, respuestas psicológicas, duelo, conocimiento de sentimientos profesionales y personales), culturales y religiosos (creencias del enfermo y cuidadores, espiritualidad, actitudes y prácticas ante la enfermedad y la muerte), éticos (trabajo en equipo) y prácticos (recursos de cuidados paliativos, integración de niveles asistenciales)

Formación de nivel intermedio

Ampliación de la formación básica de posgrado. Incluye la realización de cursos de 60-80 h y las estancias en unidades de cuidados paliativos

Formación de nivel avanzado

Realización de un curso avanzado de cuidados paliativos. Duración de 40-50 créditos docentes, de 400-500 h, y estancia en un centro de referencia

ducción de formación, categorías de priorización de la formación (aspectos clínicos y organizativos de la práctica asistencial).

- Correlaciones: entre nivel de formación y escalas tipo Likert de necesidad y demanda de formación, y entre nivel de formación y categorías de priorización de formación.

Respecto a los grupos focales, los textos se analizaron y asignaron a distintas categorías de información discutidas y consensuadas por dos investigadores. El análisis siguió la metodología de:

- Selección de fragmentos relevantes al fenómeno que se investigó.

- Selección de los ejemplos más ilustrativos de la información emergente (por saturación).

- Categorización del material de la muestra.

\section{Resultados}

Del total de 191 equipos invitados a participar (70,5\% del total del ICS), respondieron al cuestionario 132 $(69,1 \%)$

Se recogió información de 3.788 profesionales, entre MF (45,3\%), MIR (5,8\%), DUE $(45,8 \%)$ y TS (3,1\%), y se analizaron los datos de 3.568 profesionales (sin contabilizar los 220 MIR). En la tabla III se observa la descripción de los resultados de niveles de formación por categorías profesionales

En un 25\% de los EAP había algún médico con formación avanzada. En un $25 \%$ de los centros había dos o más con formación intermedia, y en un 
Tabla III. Distribución de los niveles de formación por categoría profesional.

\begin{tabular}{|c|c|c|c|c|c|c|c|c|}
\hline & \multicolumn{2}{|c|}{ MF } & \multicolumn{2}{|c|}{ DUE } & \multicolumn{2}{|c|}{ TS } & \multicolumn{2}{|c|}{ Total } \\
\hline & $n$ & $\%$ & $n$ & $\%$ & $n$ & $\%$ & $n$ & $\%$ \\
\hline Formación avanzada & 51 & 3,0 & 61 & 3,5 & 9 & 7,6 & 121 & 3,4 \\
\hline Formación intermedia & 272 & 15,9 & 246 & 14,2 & 24 & 20,3 & 542 & 15,2 \\
\hline Formación básica & 924 & 53,8 & 866 & 49,9 & 62 & 52,5 & 1.852 & 51,9 \\
\hline Subtotal & 1.247 & 72,7 & 1.173 & 67,6 & 95 & 80,5 & 2.515 & 70,5 \\
\hline Missing & 469 & 27,3 & 561 & 32,4 & 23 & 19,5 & 1.053 & 29,5 \\
\hline Total & 1.716 & 100 & 1.734 & 100 & 118 & 100 & 3.568 & 100 \\
\hline
\end{tabular}

MF: médicos de familia; DUE: diplomados universitarios de enfermería; TS: trabajador social.

Tabla IV. Necesidad, demanda y factibilidad de la formación en cuidados paliativos de los equipos de atención primaria.

\begin{tabular}{|c|c|c|c|c|c|c|c|c|}
\hline \multicolumn{3}{|c|}{$\begin{array}{c}\text { Necesidad } \\
\text { de formación }\end{array}$} & \multicolumn{3}{|c|}{$\begin{array}{c}\text { Demanda } \\
\text { de formación }\end{array}$} & \multicolumn{3}{|c|}{$\begin{array}{l}\text { Factibilidad de introducción } \\
\text { de la formación }\end{array}$} \\
\hline & $n$ & $\%$ & & $n$ & $\%$ & & $n$ & $\%$ \\
\hline Nada & 1 & 0,8 & Nada & 2 & 1,5 & Nada & 2 & 1,5 \\
\hline Necesaria & 12 & 9,1 & Poco & 28 & 21,2 & Poco & 12 & 9,2 \\
\hline Bastante & 47 & 35,6 & Demandada & 49 & 37,1 & Factible & 32 & 24,4 \\
\hline Totalmente & 72 & 54,5 & Bastante & 44 & 33,3 & Bastante & 45 & 34,4 \\
\hline \multirow[t]{2}{*}{ Total } & 132 & 100 & Totalmente & 9 & 6,8 & Totalmente & 40 & 30,5 \\
\hline & & & Total & 132 & 100 & Total & 131 & 100 \\
\hline
\end{tabular}

$50 \%$ de los centros, seis o más médicos con formación básica en cuidados paliativos.

Los porcentajes son muy similares entre DUE y en la información recogida de los médicos.

Por último, del total de TS de los que se recogió información, considerando que la media de profesionales por centro era de $1 \pm 0,6$, los resultados muestran que en un $25 \%$ de los centros había uno o más con formación básica, y en un $27 \%$ había alguno con formación avanzada o intermedia.

El total de actividades formativas dedicadas a cuidados paliativos durante 2007 contabilizadas en 128 EAP era de 256 . En un $50 \%$ de los centros el número de actividades fue de una o más, y la media fue de dos.

\section{Necesidad, demanda y factibilidad}

Respecto a las preguntas realizadas sobre la necesidad percibida, el 90,1\% de los directores de EAP de los equipos participantes percibía la formación como bastante o totalmente necesaria; el 40,1\% consideraba bastante o totalmente demandada la formación por los profesionales, y el 64,9\% veía bastante o totalmente factible la introducción de un curso de cuidados paliativos en el programa anual de formación en el momento de la recogida de información (Tabla IV).

\section{Aspectos asistenciales y organizativos priorizados}

En relación a la prioridad formativa sobre aspectos clínicos asistenciales, el 75,6\% de los centros consideró lo más o bastante prioritario el control efectivo del dolor y poco o lo menos prioritario la atención a la familia en un 53,5\% de los centros.

En relación a los aspectos organizativos, el $61 \%$ consideró lo más o bastante prioritario la toma de decisiones multidisciplinar, y el 58,8\%, el trabajo en equipo, y poco o lo menos prioritario la gestión de casos en la comunidad en un 56,5\% de los centros.

\section{Grupos focales}

Tras el análisis de los discursos de los grupos focales se identificaron puntos comunes que suponen limitaciones en la calidad asistencial que la atención primaria ofrece a los pacientes con necesidades de atención paliativa. Estas debilidades asistenciales se asocian fundamentalmente a:

- Falta de una formación integral que prepare a los profesionales de atención primaria para dar respuesta a las necesidades especificas de estos pacientes y de sus familiares y la formación que los PADES reciben para ser formadores.

- Falta de recursos en atención primaria para dar respuesta a los pacientes en situación de enfermedad avanzada.

- Falta de coordinación entre los distintos equipos/ niveles asistenciales.

A continuación se destacan aspectos específicos de la práctica asistencial de cada grupo.

\section{Profesionales de AP}

Los aspectos que generan mayor inseguridad en este colectivo en relación al manejo de pacientes paliativos se concretan en cuatro áreas fundamentales: manejo de fármacos, dimensión emocional, asistencia domiciliaria y coordinación en la gestión de casos (Tabla V). 


\section{Profesionales de PADES y expertos docentes}

La percepción de estos profesionales sobre la necesidad de formación de los profesionales de atención primaria coincide en los puntos detallados previamente. Los temas de mayor dificultad y que generan mayor necesidad formativa son: abordaje integral del paciente avanzado y su familia, manejo de aspectos emocionales, manejo farmacológico, y acceso a la formación reglada y poca demanda formativa, centrada en aspectos farmacológicos, sedación y vías subcutáneas, pero poco en aspectos de comunicación y gestión emocional (Tabla VI).

Los profesionales de PADES identifican como debilidad el impacto incierto de la formación sobre la práctica diaria. La inequidad de acceso a formación desde áreas rurales y la escasez de oferta en formación de formadores son también inquietudes presentes en los discursos.

Para ofrecer una formación de mayor calidad coinciden en destacar que ésta debe ser vivencial (estancias) y obligatoria. Se considera fundamental la revisión de los modelos docentes actuales, incluyendo las estancias o rotaciones en servicios específicos, así como el énfasis en la formación de formadores, responsables de la transmisión de conocimientos de la disciplina.

\section{Discusión}

La participación de casi el 50\% de los EAP del ICS permite concluir que los resultados obtenidos son una muestra ajustada a las necesidades de formación en cuidados paliativos de los profesionales de atención primaria de Cataluña.

Cabría destacar el alto porcentaje de profesionales con formación básica y que la cobertura de formación globalmente (básica + intermedia + avanzada) supera el $70 \%$ de profesionales.

Como se ha visto en la tabla III, el porcentaje en el nivel de formación es muy similar entre los tres colectivos estudiados.

Respecto a las necesidades de formación, se percibe como necesaria y factible de introducirse en los programas anuales de formación, pero proporcionalmente es poco demandada.

En relación a los contenidos de la formación, ambos métodos identifican como áreas clave el control de síntomas, las habilidades comunicativas y la optimización de la coordinación asistencial, elementos clave en el currículo básico de cuidados paliativos $[16,17]$. Esta necesidad coincide con otras experiencias internacionales $[18,19]$. Sin embargo, se
Tabla V. Grupos focales: conclusiones de profesionales de atención primaria

Coordinación en la gestión de casos

El profesional de atención primaria debe ser el de referencia en la comunidad y destacan que la falta de conocimiento o desorganización en la gestión del caso ('estos problemas de coordinación hacen que no puedas atender a estos pacientes como lo necesitan'), la escasa comunicación con los equipos específicos de cuidados paliativos ('mi relación con el equipo de cuidados paliativos es sólo telefónica, no los veo nunca'), la ineficacia del sistema o la falta de recursos sociales ('los pacientes terminales y sus familias tienen unas necesidades que hay que satisfacer, y yo veo que muchas veces no contamos con los recursos sociales, por ejemplo, para dar respuestas a esas necesidades', 'los recursos sociales no llegan y eso es muy duro') son las principales limitaciones en el proceso de gestión de casos

Asistencia domiciliaria

Se hace énfasis en el control de síntomas, la prevención, el trabajo de educación con los pacientes y las familias

Dimensión emocional

Está vinculada a los procesos de información y comunicación o la prevención y manejo del duelo: 'a nosotros, por donde estamos, como no hay nadie más, siempre tenemos que asumir estos pacientes y toda la carga emocional que suponen; al principio no te hace gracia, pero vas aprendiendo y te vas acostumbrando', 'ante el manejo de las emociones, como muchas veces no nos vemos capaces, pues acabas derivando a ese paciente', 'como profesional tienes que poder ayudar a los familiares de un enfermo terminal a elaborar el duelo, pero para poder hacer eso, necesitamos que nos formen a nosotros'

Manejo de fármacos

Se relaciona con la falta de formación en su indicación y las dificultades burocráticas

Tabla VI. Grupos focales: conclusiones de profesionales de PADES.

Abordaje integral del paciente avanzado y su familia

'Lo que veo es que a menudo los profesionales de atención primaria hacen las visitas con un enfoque que no es de cuidados paliativos, sino basado en la monitorización de constantes o el control de los síntomas y poco centrado en la comunicación con el paciente y la familia'

'La clave es el modelo asistencial; es decir, nosotros tenemos un modelo multidisciplinario y abordamos muchas cuestiones de las que pueden abordar los equipos de atención primaria'

\section{Manejo de los aspectos emocionales}

'El tema de la comunicación y la gestión de las emociones es el que resulta más difícil de transmitir y de enseñar a los equipos de atención primaria porque nosotros, como profesionales que nos dedicamos a los cuidados paliativos, ni siquiera estamos suficientemente formados en estos temas'

'Aparte de las carencias en formación, también hay otro tema fundamental, que es el hecho de enfrentarse a la muerte y de hablar con el paciente y la familia; es una cosa que a los equipos de atención primaria les da mucho miedo y por eso no abordan el tema y te piden que lo hagas tú

Manejo farmacológico

'Habría que hacer formación en control de síntomas, para que los equipos de atención primaria sean capaces de enfrentarse a ello de manera eficaz, es algo básico'

Acceso a la formación reglada y poca demanda formativa

'Lo que nos queda claro en el día a día, por lo que vemos nosotros, es que falta mucha formación de cuidados paliativos en atención primaria'

'Y algunos te lo dicen claramente, que tienen problemas de acceso a formación en cuidados paliativos' 'Por mi experiencia, tengo la sensación de que por parte de los equipos de atención primaria tampoco hay una demanda clara de formación; supongo que en parte también es por desconocimiento, porque si no conoces todo lo que hay, pues no lo pides'

'Siempre nos piden lo mismo y cosas muy concretas, cosas que les hemos explicado anteriormente'

destacan poco los aspectos de evaluación integral, fundamental en cuidados paliativos, o los aspectos éticos, frecuentes en domicilio [20]. 
Los directores de EAP destacan un área de mejora en una práctica poco frecuente entre los EAP, la toma de decisiones multidisciplinares, así como el énfasis en la coordinación asistencial, elemento destacado también en los grupos focales, junto al manejo de fármacos y la dimensión emocional.

Los grupos focales destacan la baja demanda formativa en aquellos temas más relevantes en la atención a pacientes en situación terminal, como la atención integral paliativa o la comunicación.

No se hace mención a la formación en planificación de las decisiones anticipadas, aspecto relevante en el proceso de toma de decisiones compartidas y evaluación de expectativas, valores y preferencias $[21,22]$, especialmente en situaciones de deterioro y dependencia de larga evolución, como es el caso de las demencias.

La metodología docente se cuestiona. Se hace poca referencia a la evaluación del impacto de la formación en la mejora de la calidad asistencial de los pacientes y sus familias, siendo un indicador principal en las líneas docentes [23].

Observamos que la oferta formativa en cuidados paliativos en Cataluña es mejorable. Algunas sociedades científicas [24-26] proponen acciones formativas enfocadas al paciente avanzado, pero se restringe a un colectivo profesional y a niveles básicos. La formación más avanzada acostumbra a estar muy dirigida a profesionales de equipos específi$\cos [27,28]$.

Gracias a este estudio hemos podido constatar que las necesidades reales de los profesionales de atención primaria existen y coinciden con las percibidas por los profesionales docentes y expertos. Se relacionan con temas muy bien identificados y claves para garantizar un modelo de atención de calidad.

Para dar respuestas a estas necesidades y mejorar la satisfacción de profesionales y pacientes [29], es necesaria la regularización de la formación, estableciendo niveles de cobertura y de contenidos adaptados a cada contexto y nivel asistencial. De ahí la importancia del desarrollo de planes formativos internos (intraequipos) que contemplen aspectos de cuidados paliativos, así como la integración de planes formativos en sistemas de pregrado, posgrado e instituciones sanitarias de relevancia, que atienden con frecuencia pacientes con necesidades paliativas (servicios de atención primaria, medicina interna, neumología, cardiología, etc.).

Estos planes deberán ofrecer contenidos docentes homogéneos, reforzando aspectos de control de síntomas, emocionales y de comunicación, ética clínica, planificación de las decisiones anticipadas [30] y gestión de caso, así como formación de for- madores, que garantice una formación de máxima calidad.

Por último, creemos imprescindible acompañar la formación de sistemas de evaluación que midan su impacto en la asistencia directa al paciente y a partir de la satisfacción del paciente, su familia y también del profesional.

La relevancia de este estudio se centra en la coincidencia de los resultados comparados entre ambas metodologías, lo que permite definir unas conclusiones extrapolables a la población sanitaria comunitaria, no sólo de Cataluña, sino de otros sistemas sanitarios. Esto permite sentar las bases de una formación ajustada a las necesidades, homogénea y promotora de la calidad asistencial y la satisfacción de los pacientes y sus familias.

A pesar de que la muestra es representativa estadísticamente, sólo se ha estudiado a los profesionales del ICS. La información obtenida de los profesionales pasó el filtro de los directores de equipo, considerando como un posible sesgo la variabilidad en la recogida de información de los profesionales de sus equipos. La muestra de los grupos focales fue menor de la planificada, por lo que la información puede ser parcial.

\section{Bibliografía}

1. Sociedad Española de Cuidados Paliativos (SECPAL). Directorio de cuidados paliativos. URL: http://www.secpal. com/directorio/index.php. [18.08.2011].

2. Espinosa J, Ela S, Beas E, Gómez-Batiste X. Directori de cures pal-liatives de Catalunya. DirCat-CP 2010 URL: http://ico.gencat.cat. [18.08.2011].

3. Gómez-Batiste X, Paz S. Public palliative care. Current Opinion in Supportive \& Palliative Care 2007; 1: 213-7.

4, Gómez-Batiste X, Fontanals MD, Roca J, Borràs JM, Viladiu P, Stjernswärd J, et al. Catalonia WHO demonstration project on palliative care implementation 1990-1995: results in 1995. J Pain Symptom Manage 1996; 12: 73-8.

5. Gómez-Batiste X, Porta-Sales J, Pascual A, Nabal-Vicuña M, Espinosa-Rojas J, Paz S, et al. Catalonia WHO palliative care demonstration at 15 years (2005). J Pain Symptom Manage 2007; 33: 584-90.

6. Gómez-Batiste X, Nabal-Vicuña M, Trelis-Navarro J, Roca-Casas J, Porta-Sales J, Paz-Ruiz S, et al. Evaluación cualitativa proyectos OMS CPs Cataluña 15 años. Med Pal 2008; 15: 293-300.

7. Gómez-Batiste X, Caja C, Espinosa J, Bullich I, MartínezMuñoz M, Porta-Sales J, et al. The Catalonia World Health Organization demonstration project for palliative care implementation: quantitative and qualitative results at 20 years. J Pain Symptom Manage 2012; 43: 783-94.

8. Gómez-Batiste X, Porta-Sales J, Paz S, Stjernsward J. Palliative medicine: models of organization. In Walsh D, ed. Palliative medicine. Philadelphia: Elsevier; 2008. p. 23-9.

9. Gómez-Batiste X, Paz S, Porta-Sales J, Rocafort J. Program development: palliative medicine and public health services. In Walsh D, ed. Palliative medicine. Philadelphia: Elsevier; 2008. p. 198-202.

10. Ministerio de Sanidad y Consumo. Estrategia en cuidados paliativos del Sistema Nacional de Salud. URL: http://www. msc.es/organizacion/sns/planCalidadSNS/pdf/excelencia/ 
cuidadospaliativos-diabetes/cuidados_paliativos/ estrategiaCuidadosPaliativos.pdf. [18.08.2011].

11. Departament de Salut, Generalitat de Catalunya. Planificació i avaluació. Pla director sociosanitari 2006. URL: http://www. gencat.cat/salut/depsalut/pdf/pd_socisanitari271006.pdf. [17.07.2011].

12. Sociedad Española de Cuidados Paliativos (SECPAL). Recomendaciones básicas sobre formación en cuidados paliativos. Med Pal 2007; 7: 23-5.

13. Recomendación Rec (2003) 24 del Comité de Ministros de los estados miembros sobre organización de cuidados paliativos. Madrid: Agencia de Calidad del Sistema Nacional de Salud. Ministerio de Sanidad y Consumo; 2001.

14. Hauer J, Quill T. Educational needs assessment, development of learning, objectives, and choosing a teaching approach. J Pall Med 2011; 14: 503-8.

15. Ury WA, Reznich CB, Weber CM. A needs assessment for a palliative care curriculum. J Pain Symptom Manage 2000; 20: 408-16.

16. European Association for Palliative Care. Curriculum in palliative care for undergraduate medical education. Recommendations of the EAPC; 2007. URL: http://www. eapcnet.eu. [18.08.2011].

17. Core Competences for End of Life Care. Training for health and social care staff. National End of Life Programme. NHS. 2011. URL: http://www.endoflifecareforadults.nhs.uk/ education-and-training/corecompetences. [18.08.2011]

18. Becker G, Momm F, Deibert P, Xander C, Gigl A, Wagner B, et al. Planning training seminars in palliative care: a crosssectional survey on the preferences of general practitioners and nurses in Austria. BMC Medl Educ 2010; 10: 43.
19. Mitchell G, Reymond E, McGrath B. Palliative care promoting general practice participation MJA 2004; 180: 207-8.

20. Orozco-Beltrán D, Ollero-Baturone M. Conferencia nacional para la atención al paciente con enfermedades crónicas. Aten Primaria 2011; 43: 165.

21. Boyd K, Mason B, Kendall M, Barclay S, Chinn D, Thomas K, et al. Advance care planning for cancer patients in primary care: a feasibility study. Br J Gen Pract 2010; 60: e449-58.

22. Caplan GA, Meller A, Squires B, Chan S, Willett W. Advance care planning and hospital. Age Ageing 2006; 35: 581.

23. Pelayo-Álvarez M, Agra Y. Systematic review of educational interventions in palliative care for primary care physicians. Pall Med 2006; 20: 673-83.

24. Sociedad Española de Medicina Familia y Comunitaria. URL: http://semfyc.es/es/home. [11.07.2011].

25. Societat Catalana de Medicina de Família i Comunitària. URL: http://www.camfic.cat. [11.07.2011].

26. Associació d'Infermeria Familiar i Comunitària de Catalunya. URL: http://www.aificc.cat. [11.07.2011].

27. Sociedad Española de Cuidados Paliativos. URL: http://www. secpal.com. [11.07.2011].

28. Unidad de Formación y Docencia. Institut Català d'Oncologia. URL: http://ico.gencat.cat. [30.06.2011].

29. Farber NJ, Urban SY, Collier VU, Metzeger M, Weiner J, Gil-Boyer E. Frequency and perceived competence in providing palliative care to terminally ill patients: a survey of primary care physicians. J Pain Symptom Manage 2004; 28: 364-72.

30. Detering KM, Hancock AD, Reade MC, Silvester W. The impact of advance care planning on end of life care in elderly patients: randomized controlled trial. BMJ 2010; 340: c1345. 